\title{
Mémoire épisodique dans la maladie d'Alzheimer
}

> Les troubles de mémoire dans la maladie d'Alzheimer touchent très tôt la remémoration consciente, souvenir d'un événement et de son contexte. Pour ce qui est de la familiarité, reconnaissance sans souvenir du contexte, les études vont dans le sens tantôt d'une altération précoce, tantôt d'une préservation de la familiarité dans les stades légers et modérés de la maladie d'Alzheimer. Ce manque de consensus peut, en partie, être expliqué par des différences méthodologiques entre les études et révèle le caractère complexe de cette forme de mémoire qui serait sous-tendue par une multitude de mécanismes pouvant être sélectivement altérés ou préservés au cours de la maladie. <

\section{La maladie d'Alzheimer et le trouble cognitif léger}

La maladie d'Alzheimer est une maladie neurodégénérative, d'apparition insidieuse, qui entraîne une dégradation progressive et globale des fonctions cognitives. Dans sa forme typique, la maladie d'Alzheimer débute généralement par une atteinte précoce de la mémoire épisodique (ou souvenir des événements personnellement vécus dans un contexte spatio-temporel particulier), qui s'étend à d'autres domaines de la cognition, et dont la sévérité est telle que la réalisation des activités de la vie quotidienne s'en trouve perturbée.

Un intérêt tout particulier est actuellement porté sur les difficultés précoces observées chez les personnes qui présentent un trouble cognitif léger [31]. Le trouble cognitif léger est un syndrome intermédiaire entre les changements cognitifs observés au cours du vieillissement normal et ceux observés dans les maladies neurodégénératives. Le trouble cognitif léger se définit par une détérioration, supérieure à celle qui est observée dans le vieillissement normal, d'une ou

Dans cette synthèse, nous utiliserons remémoration consciente dans le sens du terme anglais « recollection».

\section{Déclin de la remémoration consciente et de la familiarité ?}

Jessica Simon, Christine Bastin

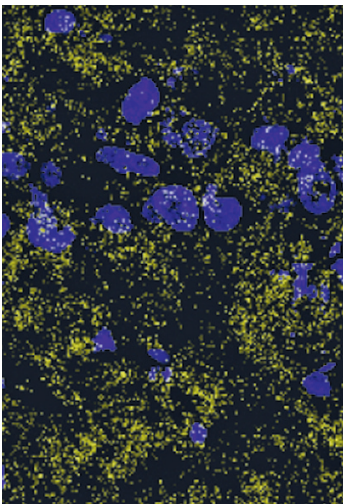

${ }^{1}$ Centre de Recherches du Cyclotron, université de Liège, allée du 6 août, B30, 4000 Liège, Belgique. j.simon@ulg.ac.be christine.bastin@ulg.ac.be

plusieurs fonctions cognitives, sans altération du niveau d'efficience cognitive globale et en l'absence de perturbation significative des activités de la vie quotidienne. Selon R.C. Petersen [1], différents sous-types de trouble cognitif léger peuvent être identifiés en fonction du domaine cognitif altéré. D'une part, le trouble cognitif léger de type amnésique regroupe les patients présentant une altération de la mémoire épisodique et, d'autre part, le trouble cognitif léger de type non amnésique regroupe les patients présentant une altération cognitive autre que mnésique. Ces deux sous-types peuvent encore être subdivisés en fonction du nombre de domaines cognitifs altérés (un ou plusieurs). On sait aujourd'hui que ces différents sous-types de trouble cognitif léger n'évoluent pas vers les mêmes entités démentielles. Dans la mesure où une grande proportion des patients souffrant d'un trouble cognitif léger amnésique évoluent vers la maladie d'Alzheimer, celui-ci est considéré comme la phase prodromale de la maladie d'Alzheimer [2]. Dans ce cadre, nous pouvons citer les critères diagnostiques révisés de Dubois et al. [3] pour la maladie d'Alzheimer au stade prodromal. Pour parler de maladie d'Alzheimer probable, une atteinte précoce et progressive de la mémoire épisodique (en association ou non avec d'autres altérations cognitives) doit être rapportée depuis plus de six mois par le patient ou un informant, et objectivée lors d'un test neuropsychologique. La certitude du diagnostic peut être améliorée en ayant recours à l'utilisation de biomarqueurs (IRM [imagerie par résonance magnétique], PET [positron emission tomography], CSF [cerebrospinal fluid]) ou en recherchant la présence d'une mutation autosomique dominante familiale. 
Les données épidémiologiques rapportent un taux de conversion annuel moyen vers une maladie d'Alzheimer de 10 à $20 \%$ pour les patients présentant un trouble cognitif léger amnésique, alors qu'il serait de 1 à $2 \%$ dans la population normale $[4,32]$. Au vu de la forte probabilité de développer une maladie d'Alzheimer dans cette population, il est clair que le dépistage précoce du trouble cognitif léger amnésique devient un enjeu majeur de santé publique. Afin de développer des outils de diagnostic sensibles et spécifiques, il est nécessaire de caractériser précisément la nature des difficultés de mémoire observées dans le trouble cognitif léger amnésique, en référence aux modèles actuels du fonctionnement de la mémoire épisodique.

\section{La remémoration consciente et la familiarité}

Selon les modèles classiques en deux processus de reconnaissance [5], deux fonctions distinctes seraient impliquées dans la récupération d'une information en mémoire à long terme. D'une part, la remémoration consciente fait référence à la récupération consciente et contrôlée d'une information en lien avec son contexte spatio-temporel d'apprentissage. Par exemple, lorsqu'on rencontre un individu que l'on connaît dans la rue, on peut se rappeler l'endroit où on l'a rencontré. D'autre part, la familiarité fait référence à une fonction relativement automatique de récupération de l'information sans accès conscient au contexte spatio-temporel d'apprentissage. Par exemple, on croise une personne que l'on reconnaît dans la rue sans pouvoir se rappeler qui elle est ou à quelle occasion on l'a rencontrée.

La recherche en neurosciences cognitives suggère que ces deux formes de mémoire sont indépendantes, reposent sur des réseaux cérébraux partiellement distincts et peuvent être dissociées dans certaines populations. Ainsi, un déclin sélectif de la remémoration consciente, accompagné d'une préservation de la familiarité, a été décrit lors du vieillissement normal ou chez des patients devenus amnésiques suite à une atteinte de l'hippocampe. Les données actuelles soulignent la contribution des régions hippocampiques et para-hippocampiques dans la remémoration consciente, alors que la familiarité serait sous-tendue par le cortex périrhinal [6]. La suite de cette synthèse examinera si la maladie d'Alzheimer et le trouble cognitif léger amnésique affectent sélectivement l'une de ces formes de mémoire ou les touchent toutes les deux.

\section{Impact de la maladie d'Alzheimer et du trouble cognitif léger amnésique sur la remémoration consciente et la familiarité}

Il est aujourd'hui bien établi que la fonction de remémoration consciente est altérée très tôt dans la maladie d'Alzheimer, dès le stade du trouble cognitif léger [7-9]. En revanche, pour ce qui est de la familiarité, certaines études apportent des données en faveur de sa préservation dans les stades légers et modérés de la maladie d'Alzheimer [7, 9-11], alors que d'autres suggèrent une altération très précoce $[12,13]$. Ce manque de consensus peut s'expliquer, au moins en partie, par des différences méthodologiques entre les études. Nous verrons que celles-ci révèlent que la familiarité est une fonction com- plexe, dont certaines facettes seraient plus touchées par la pathologie neurodégénérative que d'autres. Nous aborderons les études existantes en fonction de trois dimensions méthodologiques: le type de paradigme de reconnaissance, le type de paradigme d'estimation de la familiarité et de la remémoration consciente, et la nature du matériel utilisé.

\section{Méthode}

Pour cette revue de la littérature, nous n'avons sélectionné que les articles traitant de l'évaluation de la remémoration consciente et de la familiarité chez des patients souffrant d'un trouble cognitif léger amnésique ou d'une maladie d'Alzheimer, indépendamment de l'âge et du sexe, parus après 2000 et rédigés en français ou en anglais. II n'y a pas eu de restriction quant à la méthode utilisée pour le diagnostic de ces deux groupes de patients. Nous avons inclus des données issues d'études comportementales et par imagerie cérébrale. Pour ce faire, nous avons fait une recherche sur Psychlnfo (OvidSP), ainsi que sur Scopus (SciVerse). Pour PsychInfo, les descripteurs étaient les suivants: (Alzheimer's disease or mild cognitive impairment.mp) and (recognition [learning] or familiarity or recollection.mp or remember.mp). Pour Scopus, nous avons utilisé les mots-clés suivants: (Alzheimer or mild cognitive impairment) and (recollection or familiarity or process-dissociation procedure) dans les champs article, titre, abstract ou mots-clés.

\section{Le type de paradigme de reconnaissance}

Les tâches de reconnaissance consistent à présenter au participant une série d'items (phase d'encodage), puis à lui demander ultérieurement de discriminer les items vus de ceux qu'il n'a pas vus (phase de reconnaissance). Deux grands paradigmes de reconnaissance peuvent être identifiés. Premièrement, les tâches de reconnaissance « à choix forcé » : le participant doit déterminer, parmi plusieurs items présentés simultanément, celui qui a été encodé précédemment. Deuxièmement, les tâches de reconnaissance «oui/non » : le participant doit indiquer pour chaque item présenté séquentiellement s'il a été encodé précédemment ou non.

Analysée dans le cadre des théories en deux processus et bien que la remémoration consciente et la familiarité puissent toutes deux contribuer aux performances, la réalisation de tests de reconnaissance « oui/non » ferait intervenir davantage la mise en place d'un processus de remémoration consciente que les tests «à choix forcé ». Ceux-ci, en revanche, pourraient être réussis en comparant le sentiment de familiarité associé à chacun des items présents et en sélectionnant celui qui semble le plus familier [14]. 
Les données actuelles sont en faveur d'une altération des performances en reconnaissance pour les tâches de type «oui/non » et d'une préservation des performances dans les tâches de reconnaissance «à choix forcé » dans le trouble cognitif léger amnésique. Chez les patients souffrant de la maladie d'Alzheimer, en revanche, les données vont dans le sens d'une altération des performances pour les deux types de tâches $[15,16]$. Ces résultats pourraient suggérer une atteinte préférentielle de la remémoration consciente dès les premiers stades de la maladie d'Alzheimer, alors que la familiarité ne serait altérée que plus tardivement. Sur le plan diagnostique, selon Clark et al. [15], le déficit observé dans les tâches de reconnaissance « oui/non » serait un bon indicateur de la présence de la pathologie d'Alzheimer, même à des stades très précoces, alors que le déficit observé lors des tâches de reconnaissance «à choix forcé » serait un indicateur de l'évolution de la pathologie, et permettrait de différentier les patients avec trouble cognitif léger amnésique des patients avec maladie d'Alzheimer. Cependant, ces deux procédures ne mesurent pas explicitement la familiarité et la remémoration consciente. Elles n'apportent donc que des preuves indirectes de la contribution de ces deux fonctions lors d'une tâche de mémoire. Différents paradigmes ont été spécifiquement développés pour répondre à ce problème.

\section{Les paradigmes d'estimation de la familiarité} et de la remémoration consciente

Nous aborderons dans cette section quatre paradigmes bien connus d'estimation de la contribution des processus de reconnaissance (Tableau I).

- Tout d'abord, le paradigme «remember/know » [17] repose sur l'évaluation, faite par l'individu, de l'expérience subjective associée à la reconnaissance d'une information. Lors d'une tâche de reconnaissance, il est

\begin{tabular}{|c|c|c|c|c|c|c|c|}
\hline \multirow[b]{2}{*}{ Études } & \multicolumn{3}{|c|}{ Groupes* } & \multirow[b]{2}{*}{ Paradigmes } & \multirow[b]{2}{*}{ Stimulus } & \multirow{2}{*}{$\begin{array}{l}\text { Altération de } \\
\text { remémoration } \\
\text { consciente }\end{array}$} & \multirow{2}{*}{$\begin{array}{l}\text { Altération } \\
\text { de familiarité }\end{array}$} \\
\hline & $\begin{array}{l}\text { Âgés } \\
\text { sains }\end{array}$ & $\begin{array}{c}\text { Trouble } \\
\text { cognitif léger }\end{array}$ & $\begin{array}{l}\text { Maladie } \\
\text { d'Alzheimer }\end{array}$ & & & & \\
\hline [21] & 16 & 16 & & Courbes ROC & Images et mots & oui & Images: non ; mots : oui \\
\hline [13] & 12 & 11 & 10 & Courbes ROC & Mots & oui & oui \\
\hline$[25]$ & 17 & 14 & & Items vs associations & Images & oui & oui \\
\hline [11] & 17 & & 32 & Items vs associations & Mots & oui & non \\
\hline [12] & 50 & 32 & & Items vs associations & Mots & oui & oui \\
\hline [27] & 12 & & 12 & Items vs associations & Mots & oui & oui \\
\hline [25] & 21 & 16 & & Items vs associations & Mots & oui & oui \\
\hline [9] & 23 & 19 & & PDP & Mots & Marginale & non \\
\hline [23] & 45 & 17 & & PDP & Mots & oui & non \\
\hline$[26]$ & 10 & & 17 & PDP & Mots & oui & non \\
\hline [26] & 10 & & 10 & PDP & Mots & oui & oui \\
\hline$[7]$ & 21 & 23 & 27 & PDP & Mots & oui & non \\
\hline [25] & 18 & 13 & & PDP & Mots & oui & oui \\
\hline [9] & 23 & 19 & & Remember/know & Mots & oui & non \\
\hline [10] & 21 & & 21 & Remember/know & Mots & oui & non \\
\hline [19] & 10 & & 13 & Remember/know & Mots & oui & non \\
\hline [8] & 23 & 20 & 10 & Remember/know & Mots & oui & $\begin{array}{l}\text { Trouble cognitif léger : non } \\
\text { Maladie d'Alzheimer : oui }\end{array}$ \\
\hline [18] & 30 & 31 & & Remember/know & Visages & oui & non \\
\hline
\end{tabular}

Tableau I. Altération de la remémoration consciente et de la familiarité en fonction du paradigme de reconnaissance et de la nature des stimulus. * Nombre de sujets par groupe; PDP : procédure de dissociation des processus ; items vs (versus) associations : paradigme de comparaison entre la mémoire pour des items individuels et la mémoire pour des associations. 
demandé aux participants de déterminer si la reconnaissance d'un item se base sur la récupération de détails liés au contexte d'apprentissage (réponse remember, correspondant à une expérience de remémoration consciente) ou sur un sentiment de familiarité sans récupération de détails contextuels liés à l'encodage (réponse know). Les études ayant utilisé ce paradigme dans le trouble cognitif léger amnésique $[8,9$, 18] et dans la maladie d'Alzheimer [10, 19] tendent à suggérer que l'expérience de remémoration consciente est altérée, alors que le sentiment de familiarité serait préservé dans les deux populations. À notre connaissance, une seule étude a rapporté des données en faveur d'une altération du sentiment de familiarité dans la maladie d'Alzheimer [8].

- Un second paradigme est celui qui permet le calcul de courbes «receiver operating characteristics » ou courbes ROC [20]. Typiquement, lors d'un test de reconnaissance, les participants doivent émettre un jugement quant à la certitude de leurs réponses sur une échelle en plusieurs points (généralement en six points allant de « 1 = certain que nouveau » à $« 6$ = certain qu'ancien $»)$. En fonction des proportions de réponses correctes et incorrectes à chaque niveau de confiance, il est possible d'inférer la contribution de la remémoration consciente et de la familiarité. En pratique, la remémoration consciente est donnée par l'ordonnée à l'origine de la courbe ROC, et la familiarité par sa curvilinéarité. Les études ayant utilisé ce paradigme sont relativement peu nombreuses. Ally et al. [13] ont rapporté une altération de la remémoration consciente et de la familiarité dans la maladie d'Alzheimer et le trouble cognitif léger amnésique, alors que Embree et al. [21] ont rapporté une altération de la remémoration consciente et une préservation de la familiarité pour du matériel visuel uniquement (et pas pour du matériel verbal) dans le trouble cognitif léger amnésique.

- Un troisième paradigme est la procédure de dissociation des processus développée par Jacoby [22]. Ce paradigme permet de dissocier la contribution des processus conscients et contrôlés sous-tendant la remémoration consciente, de la contribution de processus plus inconscients et automatiques sous-tendant la familiarité. Pour ce faire, l'expérimentateur crée deux conditions de récupération au sein de la même tâche: une condition d'inclusion et une condition d'exclusion. Ces conditions ont été appliquées principalement à des tâches de complétion de mots et des tâches de discrimination contextuelle. Dans la condition d'inclusion des tâches de complétion de mots, il est demandé aux participants de compléter un trigramme représentant le début d'un mot avec un mot étudié au préalable ou avec le premier mot qui leur vient à l'esprit. Dans la condition d'exclusion, il leur est demandé de compléter le trigramme avec un mot qui n'a pas été étudié préalablement. Dans les tâches de discrimination contextuelle, les items sont encodés en lien avec un élément contextuel comme une couleur (vert ou rouge). Lors de la tâche de reconnaissance, dans la condition d'inclusion, il est demandé aux participants de répondre oui pour tous les items présentés antérieurement. Dans la condition d'exclusion, il est demandé aux participants de répondre oui si l'item a été présenté à l'encodage sur un fond de couleur rouge, et de répondre non si l'item a été encodé sur un fond d'une autre couleur ou si l'item est nouveau. Un succès lors de la condition d'inclusion de ces deux tâches peut faire intervenir aussi bien la remémoration consciente que des processus de récupération automatique.
En revanche, un succès dans la condition d'exclusion requiert nécessairement l'utilisation de la remémoration consciente, qui permet le rappel des items en lien avec leur contexte d'apprentissage. En cas d'échec, on suppose que la réponse fait intervenir des processus de récupération automatique. Les études ayant utilisé ce paradigme mettent le plus souvent en évidence un déficit de la récupération contrôlée et une relative préservation des processus de récupération automatique dans le trouble cognitif léger amnésique $[7,23]$ et dans la maladie d'Alzheimer $[7,24]$, bien que certaines études aient rapporté une altération, parfois très subtile, des processus de récupération automatique dans le trouble cognitif léger [25] et dans la maladie d'Alzheimer [26]. - Enfin, on peut citer le paradigme de comparaison entre la mémoire pour des items individuels et la mémoire pour des associations. Ce paradigme repose sur le postulat que la fonction de remémoration consciente sous-tendrait la récupération d'associations créées à l'encodage, alors que la reconnaissance d'un item isolé pourrait être sous-tendue à la fois par la familiarité et la remémoration consciente. Une procédure typiquement utilisée consiste à présenter aux participants une série de paires (par exemple, deux mots) qu'ils doivent apprendre. Lors du test de reconnaissance, les participants doivent identifier les paires intactes (items encodés au sein de la même paire) et rejeter les paires recombinées (paires comprenant deux items encodés séparément lors de l'encodage) ou nouvelles. Le rejet d'une paire recombinée nécessite donc la récupération consciente de l'association originale. En cas d'altération de la remémoration consciente, les participants devraient présenter des difficultés pour considérer les paires recombinées comme des paires nouvelles. L'acceptation d'une paire intacte, en revanche, peut soit être sous-tendue par le rappel conscient de l'association, soit par un sentiment de familiarité vis-à-vis de chacun des deux items. La plupart des études ayant utilisé ce paradigme ont rapporté une altération de la familiarité et de la remémoration consciente dans le trouble cognitif léger amnésique [25] ou dans la maladie d'Alzheimer [12, 27]. À l'inverse, Genon et al. [11] ont rapporté une altération de la remémoration consciente associée à une préservation de la familiarité chez des sujets atteints d'une forme légère de maladie d'Alzheimer.

Prises ensemble, toutes ces données confortent l'idée d'une altération de la remémoration consciente dans la maladie d'Alzheimer et dans le trouble cognitif léger. En revanche, la divergence des résultats concernant la familiarité est compatible avec l'idée que la familiarité est une fonction complexe sous-tendue par une multitude de mécanismes. Il est important de garder à l'esprit que 
les différents paradigmes présentés ici ne mesurent pas tous les mêmes aspects de la familiarité. Ainsi, les études ayant utilisé le paradigme «remember/know » et la procédure de dissociation des processus suggèrent une relative préservation dans la maladie d'Alzheimer débutante du sentiment subjectif de familiarité et de la récupération automatique de l'information en mémoire. Quant aux études ayant utilisé la technique des courbes ROC et le paradigme de comparaison entre la mémoire de l'item et la mémoire des associations, elles apporteraient des éléments en faveur d'une altération précoce des processus de discrimination entre les cibles et les leurres, d'une part, et de la capacité à extraire les informations individuelles au sein d'une association, d'autre part.

\section{La nature du matériel utilisé en reconnaissance}

L'impact du type de matériel utilisé est une autre variable importante à prendre en compte lorsqu'on veut évaluer l'intégrité de la familiarité dans la maladie d'Alzheimer. La méthodologie des différentes études révèle la multiplicité du matériel utilisé, tel que des mots, des sons, des dessins d'objets, des photographies d'objets, des visages ou des scènes complexes. D'une manière générale, les travaux sur la reconnaissance dans la maladie d'Alzheimer et le trouble cognitif léger tendent à montrer une préservation de la familiarité lorsque les tâches utilisent du matériel visuel par rapport à du matériel verbal (pour une revue voir [28]). Ceci fait écho à l'effet de supériorité des images sur les mots, connu en psychologie cognitive, et qui se caractérise par de meilleures performances en rappel et en reconnaissance [29], ainsi que par une augmentation des réponses remember, pour du matériel imagé par rapport à du matériel verbal, que ce soit chez des participants jeunes [29] ou âgés [30]. Dans le trouble cognitif léger amnésique, Embree et al. [21] ont même montré que l'effet de supériorité des images était plus important chez les patients que chez les participants âgés. Leur interprétation de ce résultat est que l'altération de la remémoration consciente obligerait les patients avec trouble cognitif léger amnésique à devenir sur-dépendants de la familiarité lors des tâches de reconnaissance. En mesurant la familiarité associée aux items visuels et verbaux au moyen des courbes ROC, ils ont en effet montré que l'effet de supériorité des images était dû à une préservation de la familiarité pour les stimulus visuels, alors que celle-ci serait altérée pour du matériel verbal. Ces données suggèreraient une atteinte différentielle de la familiarité en fonction du matériel.

\section{Conclusion}

Actuellement, les chercheurs s'accordent sur l'idée d'une altération précoce de la fonction de remémoration consciente dans la maladie d'Alzheimer. En revanche, il n'y a pas de consensus quant à la préservation ou non de la familiarité. La diversité des résultats est compatible avec l'idée que la familiarité est une fonction complexe sous-tendue par différents mécanismes pouvant être sélectivement altérés au cours de la maladie. Le sentiment subjectif de familiarité et la récupération automatique de l'information en mémoire seraient préservés, alors que les processus de discrimination entre les cibles et les leurres, et la capacité à extraire les informations individuelles au sein d'une association seraient altérés. Les recherches futures devraient différencier les aspects de la familiarité qui sont mesurés au sein de chaque tâche, modéliser leurs interactions et poser des hypothèses précises quant à leurs altérations au cours de la maladie d'Alzheimer. Ces hypothèses pourront être testées grâce à des études longitudinales dans lesquelles des patients présentant un trouble cognitif léger amnésique seraient suivis dès l'apparition des premiers symptômes jusqu'à l'installation de la maladie d'Alzheimer.

Enfin, la question de l'altération de la familiarité est importante en clinique et, plus spécialement, dans le diagnostic précoce de la maladie d'Alzheimer. En effet, considérant que l'altération de la remémoration consciente n'est pas spécifique à la maladie d'Alzheimer, une altération d'un (ou plusieurs) mécanisme(s) sous-tendant la familiarité dès les premiers stades de la maladie, que l'on n'observerait que dans cette population, pourrait(ent) constituer un biomarqueur cognitif robuste de la pathologie au même titre que les biomarqueurs cérébraux. $\diamond$

\section{SUMMARY}

Episodic memory in Alzheimer's disease:

decline in recollection and familiarity?

According to the dual-process recognition models, the retrieval of information from long-term memory is supported by recollection and familiarity. Alzheimer's disease is consistently found to affect recollection. As for familiarity, however, no consensus has been reached so far. Some studies are in favor of an early impairment of familiarity, while others are in favor of a preservation of familiarity in the mild and moderate stages of the disease. This lack of consensus can be partly explained by methodological differences between studies. We discuss three methodological dimensions: the type of recognition test, the type of paradigm for estimating familiarity and recollection, and the nature of the material used. These differences reveal the complex nature of familiarity which would be underlied by different mechanisms that may be selectively altered or preserved in the disease. $\diamond$

\section{REMERCIEMENTS}

Ce travail a été soutenu par le Fonds pour la recherche en sciences humaines et le Fonds national pour la recherche scientifique (FRESH/FRS-FNRS), la Fondation pour la recherche Alzheimer (SAO-FRA) et la Fondation Roi Baudouin.

\section{LIENS D'INTÉRÊT}

Les auteurs déclarent n'avoir aucun lien d'intérêt concernant les données publiées dans cet article. 


\section{RÉFÉRENCES}

1. Petersen RC. Mild cognitive impairment as a diagnostic entity. J Intern Med 2004 ; 256 : 183-94

2. Albert MS, DeKosky ST, Dickson D, et al. The diagnosis of mild cognitive impairment due to Alzheimer's disease: recommendations from the National institute on aging-Alzheimer's association workgroups on diagnostic guidelines for Alzheimer's disease. Alzheimer Dement 2011 ; $7: 270-9$

3. Dubois B, Feldman HH, Jacova C, et al. Research criteria for the diagnosis of Alzheimer's disease: revising the NINCDS-ADRDA criteria. Lancet Neurol 2007 ; 6 : 734-46.

4. Ritchie K. Mild cognitive impairment: An epidemiological perspective. Dialogues Clin Neurosci $2004 ; 6: 401-8$

5. Yonelinas AP. The nature of recollection and familiarity: a review of 30 years of research. J Mem Lang $2002 ; 46: 441-517$.

6. Eichenbaum H, Yonelinas AP, Ranganath $\mathrm{C}$. The medial temporal lobe and recognition memory. Annu Rev Neurosci 2007 ; 30 : 123-52

7. Bastin C, Kerrouche N, Lekeu F, et al. Controlled memory processes in questionable Alzheimer's disease: a view from neuroimaging research. J Alzheimer Dis $2010 ; 20: 547-60$.

8. Hudon C, Belleville S, Gauthier S. The assessment of recognition memory using the remember/ know procedure in amnestic mild cognitive impairment and probable Alzheimer's disease. Brain $2009 ; 70: 171-9$.

9. Serra L, Bozzali M, Cercignani M, et al. Recollection and familiarity in amnesic mild cognitive impairment. Neuropsychology $2010 ; 24: 316-26$.

10. Genon S, Bahri MA, Collette F, et al. Cognitive and neuroimaging evidence of impaired interaction between self and memory in Alzheimer's disease. Cortex $2014 ; 51$ : 11-24.

11. Genon S, Collette F, Feyers D, et al. Item familiarity and controlled associative retrieval in Alzheimer's disease: an fMRI study. Cortex 2013; 49: 1566-84

12. Wolk DA, Mancuso L, Kliot D, et al. Familiarity-based memory as an early cognitive marker of preclinical and prodromal AD. Neuropsychologia $2013 ; 51$ : 1094-102.

13. Ally BA, Gold CA, Budson AE. An evaluation of recollection and familiarity in Alzheimer's disease and mild cognitive impairment using receiver operating characteristics. Brain $2009 ; 69: 504-13$

14. Bastin C, Van der Linden M. Une approche neuropsychologique des relations entre mémoire épisodique et mémoire sémantique. Rev Neuropsychol 2003 ; 13 : 3-69.

15. Clark LR, Stricker NH, Libon DJ, et al. Yes/No versus forced-choice recognition memory in mild cognitive impairment and Alzheimer's disease: patterns of impairment and associations with dementia severity. Clin Neuropsychol $2012 ; 26$ : 1201-16.

16. Westerberg $C \varepsilon$, Paller KA, Weintraub $S$, et al. When memory does not fail: familiarity-based recognition in mild cognitive impairment and Alzheimer's disease. Neuropsychology 2006; 20 : 193-205.

17. Tulving $\varepsilon$. Memory and consciousness. Can Psychol $1985 ; 26: 1-12$

18. Wang P, Li J, Li H, et al. Is emotional memory enhancement preserved in amnestic mild cognitive impairment? Evidence from separating recollection and familiarity. Neuropsychology 2013 ; 27 691-701.
19. Rauchs G, Piolino P, Mézenge $F$, et al. Autonoetic consciousness in Alzheimer's disease: neuropsychological and PET findings using an episodic learning and recognition task. Neurobiol Aging $2007 ; 28: 1410-20$.

20. Yonelinas AP. Receiver-operating characteristics in recognition memory: evidence for a dual-process model. J Exp Psychol Learn Mem Cogn $1994 ; 20$ : 1341-54

21. Embree LM, Budson AE, Ally BA. Memorial familiarity remains intact for pictures but not for words in patients with amnestic mild cognitive impairment. Neuropsychologia $2012 ; 50: 2333-40$.

22. Jacoby LL. A process dissociation framework: separating automatic from intentional uses of memory. J Mem Lang 1991 ; 30 : 513-41.

23. Anderson ND, Ebert PL, Jennings JM, et al. Recollection- and familiaritybased memory in healthy aging and amnestic mild cognitive impairment. Neuropsychology $2008 ; 22$ : 177-87.

24. Adam S, Van der Linden M, Collette F, et al. Further exploration of controlled and automatic memory processes in early Alzheimer's disease. Neuropsychology $2005 ; 19: 420-7$

25. Wolk DA, Signoff ED, DeKosky ST. Recollection and familiarity in amnestic mild cognitive impairment: a global decline in recognition memory. Neuropsychologia $2008 ; 46: 1965-78$

26. Smith JA, Knight RG. Memory processing in Alzheimer's disease. Neuropsychologia $2002 ; 40: 666-82$.

27. Gallo DA, Sullivan AL, Daffner KR, et al. Associative recognition in Alzheimer's disease: evidence for impaired recall-to-reject. Neuropsychology $2004 ; 18$ : 556-63.

28. Ally BA. Using pictures and words to understand recognition memory deterioration in amnestic mild cognitive impairment and Alzheimer's disease: a review. Curr Neurol Neurosci Rep 2012 ; 12 : 687-94.

29. Curran T, Doyle J. Picture superiority doubly dissociates the ERP correlates of recollection and familiarity. J Cogn Neurosci $2011 ; 23$ : 1247-62.

30. Ally BA, Waring JD, Beth $\varepsilon H$, et al. Aging memory for pictures: using highdensity event-related potentials to understand the effect of aging on the picture superiority effect. Neuropsychologia $2008 ; 46: 679-89$.

31. Dubois B, Beato R, Kalafat M. Avant la démence... ou les limites du concept de trouble cognitif léger. Med Sci (Paris) 2002 ; $18: 775-9$.

32. Sabia S, Ankri J, Singh-Manoux A. Approche vie entière dans l'étude du vieillissement cognitif. Med Sci (Paris) $2010 ; 26: 319-24$

\section{TIRÉS À PART}

J. Simon

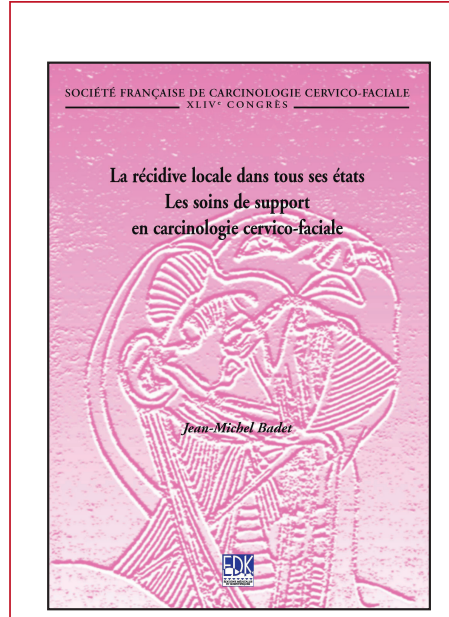

ISBN : 978-2-8425-4174-3 274 pages

\section{Bon de commande}

À retourner à EDK, 109, avenue Aristide Briand - 92541 Montrouge Cedex Tél. : 0141177405 - Fax : 0143293262 - E-mail : edk@edk.fr

NOM :

Prénom :

Adresse :

Code postal :

Ville :

Pays :

Fonction :

Je souhaite recevoir l'ouvrage La récidive locale dans tous ses états - Les soins de support en carcinologie cervico-faciale : $35 €+3 €$ de port $=\mathbf{3 8} €$ TTC

en ................. exemplaire, soit un total de ........................................ €

$\square$ Par chèque, à l'ordre de $\mathbf{E} \mathbf{D} \mathbf{K}$

$\square$ Par carte bancaire :

Visa

Carte $\mathrm{n}^{\circ}$

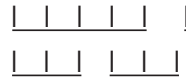

$\square$ Eurocard/Mastercard

Date d'expiration :

Signature :

$\mathrm{N}^{\circ}$ de contrôle au dos de la carte :

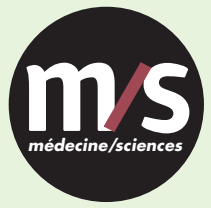

Tarifs d'abonnement $\mathrm{m} / \mathrm{s}-2014$

Abonnez-vous

à médecine/sciences
> Grâce à $m / s$, vivez en direct les progrès des sciences biologiques et médicales

Bulletin d'abonnement page 813 dans ce numéro de $\mathrm{m} / \mathrm{s}$

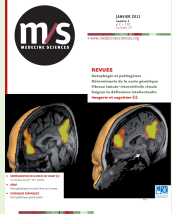

